\title{
AN EXISTENCE THEOREM FOR THE CHARACTERISTIC NUMBERS OF A CERTAIN BOUNDARY VALUE PROBLEM*
}

BY

H. T. DAVIS

The first comprehensive study of the properties of the solutions of a linear, homogeneous, self adjoint differential equation of second order,

$$
\frac{d}{d x}\left[k(x) u^{\prime}(x)\right]+g(\lambda, x) u(x)=0
$$

and a set of boundary conditions was made by Sturm in 1836.t Sturm, limiting himself to a consideration of solutions which satisfied the boundary conditions

$$
\alpha_{1} u(a)+\alpha_{2} u^{\prime}(a)=0, \quad \beta_{1} u(b)+\beta_{2} u^{\prime}(b)=0,
$$

established many fundamental theorems. One primary object was to find conditions upon the coefficients of the system sufficient to prove the existence of values of $\lambda$ to which would correspond functions satisfying both differential equation and boundary conditions. These values of $\lambda$ are called characteristic numbers. In his investigation Sturm was led to study the problem of how the zeros of these solutions depended upon the parameter and the coefficients of the system. These results have been called oscillation theorems.

Mason, $\ddagger$ restricting $g(\lambda, x)$ to the form $\lambda A(x)-B(x)$, extended Sturm's existence theorem to the case of general linear boundary conditions with constant coefficients and gave an oscillation theorem for the special case

$$
u(a)=u(b), \quad u^{\prime}(a)=u^{\prime}(b) .
$$

Bôcher, employing other means, removed Mason's restrictions on $g(\lambda, x)$ for the oscillation problem, except that a certain uniqueness of the values of $\lambda$ was not proved. $\S$

* Presented to the Society, April 15, 1922.

† Journal de Mathématiques, vol. 1 (1836), pp. 106-186.

† These Transactions, vol. 7 (1906), pp. 337-360.

$\S$ Paris Comptes Rendus, vol. 140 (1905), p. 928. 
The next advance was made by Birkhoff who gave an existence proof and an oscillation theorem for the solution $u_{p}$ of the self adjoint system of second order except that he limited himself to the case where $k=1$ and the coefficients in his boundary conditions did not vary with $\lambda$. Birkhoff's method, like that of Bôcher, was based upon an application of Sturm's theorems to this system.*

Ettlinger completed the extension to the case where $k$ varied with $\lambda$ and the coefficients of the boundary conditions were functions of $\lambda$. The essential point of Ettlinger's treatment of the problem was the introduction of a condition which pertained to the sign of a certain determinant of fourth order made from the coefficients and their $\lambda$-increments.t

In a second paper, $\ddagger$ Ettlinger used this condition in determining the oscillation properties of solutions belonging to a general set of linear boundary conditions.

The same problem with more or less generality has also been treated by Haupt $\S$ and Fort, $\|$ the latter extending his results to a corresponding system in difference equations.

The general theory underlying the methods of Sturm and a complete formulation of the boundary value problem is to be found in Bôcher's Leçons sur les Méthodes de Sturm (Paris, 1917).

The object of the present paper is, first, to develop a general method of attack for the existence problem applicable to an extension of the Sturmian problem to equations of higher order than the second and, second, to apply this method to the second order problem from which follows a simplification of both argument and conditions.

The method will be found to be a slight modification of that employed by Bôcher, Birkhoff, and Ettlinger, use being made of the same principle of comparing one system with another by means of their characteristic equations.

\section{The general PROBLem}

Suppose $L(u)$ is any linear, homogeneous, ordinary differential expression of $n$th order

$$
L(u)=p_{n}(x) \frac{d^{n} u}{d x^{n}}+p_{n-1}(x) \frac{d^{n-1} u}{d x^{n-1}}+\cdots+p_{0}(x) u
$$

* These Transactions, vol. 10 (1909), pp. 259-270.

†These Transactions, vol. 19 (1918), pp. 79-96.

$\ddagger$ These Transactions, vol. 22 (1921), pp. 136-143.

§ Dissertation, Leipzig, Teubner, 1911. Also, Math e mati s che Annalen, vol. 76 (1914), pp. 67-104.

II American Journal of Mathematics, vol. 39 (1917), pp. 1-26. 
and suppose that $p_{n}(x)$ is different from zero in the interval $a \leqq x \leqq b$. Consider. then, the two systems

$$
\begin{gathered}
L(u)=0, \\
U_{i}(u)=0,
\end{gathered}
$$

$$
L(u)=0,
$$

$$
V_{i}(u)=0 \quad(i=1,2 \ldots, n)
$$

where $U_{i}$ and $V_{i}$ are expressions at two points $a$ and $b$ of the form $\alpha_{i 1} u(a)+\alpha_{i 2} u^{\prime}(a)+\cdots+\alpha_{i n} u^{(n-1)}(a)-\beta_{i 1} u(b)-\beta_{i 2} u^{\prime}(b)-\cdots-\beta_{i n} u^{(n-1)}(b)$.

Since the general solution of the differential equation may be written in ine form

$$
u=c_{1} u_{1}+c_{2} u_{0}+\ldots+c_{n} u_{n},
$$

where the functions $u_{1}, \ldots, u_{n}$ are a fundamental set of solutions of the equation, we are led to consider the following equations in order to determine the $c_{i}$ for system I:

$$
\begin{gathered}
c_{1} U_{11}+c_{2} U_{12}+\cdots+c_{n} U_{1 n}=0, \\
c_{1} U_{21}+c_{2} U_{22}+\cdots+c_{n} U_{2 n}=0 \\
\cdot \cdot \cdot \cdot \cdot \cdot \cdot \cdot \cdot \cdot \cdot \cdot \\
c_{1} U_{n 1}+c_{2} U_{n 2}+\cdots+c_{n} U_{n n}=0
\end{gathered}
$$

where the notation $U_{i j}$ has been used to denote $U_{i}\left(u_{j}\right)$.

A similar set of conditions holds also for system II.

Consider, then, the two matrices

$$
T_{1}=\left\|\begin{array}{lll}
U_{11} & \cdots & U_{1 n} \\
\cdot & \cdot & \cdot \\
U_{n 1} & \cdots & U_{n n}
\end{array}\right\|, \quad T_{2}=\left\|\begin{array}{ccc}
V_{11} & \cdots & V_{1 n} \\
\cdot & \cdot & \cdot \\
V_{n 1} & \cdots & V_{n n}
\end{array}\right\| .
$$

The rank of a matrix is the maximum order of a determinant not zero issuing from the matrix. It is well known that if $p$ is the rank of matrix $T_{1}$, there are $n-p$ linearly independent solutions for $c_{1}, \ldots, c_{n}$. Each of these solutions gives a function $u=c_{1} u_{1}+\cdots+c_{n} u_{n}$ which satisfies system I.*

* Bôcher, Leçons, Chap. II. 
In what follows we shall suppose the ranks of both $T_{1}$ and $T_{2}$ to be $n-1$ and shall treat exceptions when they arise as special problems. We are led, thus, to consider the vanishing of two new expressions

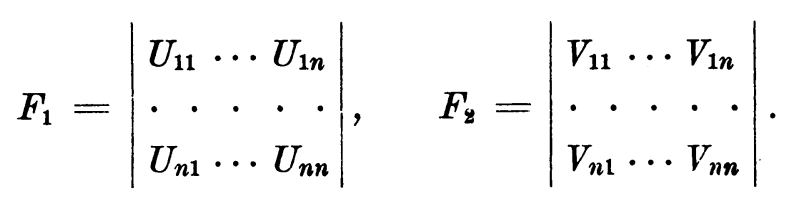

If the coefficients of $L(u), U_{i}$, and $V_{i}$ depend also upon a parameter $\lambda$, then $F_{1}$ and $F_{2}$ will be functions of $\lambda$, and for every value of $\lambda$ for which $F_{1}$ or $F_{2}$ vanishes there will correspond a solution of system I or II. The equation $F_{1}(\lambda)=0$ has been called by Bôcher the characteristic equation of system $\mathrm{I}$.

We now assume that $F_{1}(\lambda)$ is known to vanish for a succession of values $\lambda_{1}, \lambda_{2}, \ldots, \lambda_{n}$. The object of this discussion is to develop conditions under which $F_{2}$ will vanish also.

Consider the new function

$$
F(\lambda)=F_{1} F_{2}, \quad L_{1} \leqq \lambda \leqq L_{2} .
$$

If the coefficients of $L(u)$ are continuous throughout the region $a \leqq x \leqq b$, $L_{1} \leqq \lambda \leqq L_{2}$, and the coefficients of $U_{i}$ and $V_{i}$ are continuous in the interval $\left(L_{1} L_{2}\right)$, it may be shown that $F(\lambda)$ is also a continuous function in the interval $\left(L_{1} L_{2}\right){ }^{*}$ The following consequences are immediate:

Consider $F(\lambda)$ in the interval $\lambda_{i} \leqq \lambda \leqq \lambda_{i+1}$ where $\lambda_{i}$ and $\lambda_{i+1}$ are two successive zeros of $F_{1}$. If $F_{2}$ does not vanish in the interval, then the sign of $F\left(\lambda_{i}+\delta \lambda\right)$ will be the same as that of $F\left(\lambda_{i+1}-\delta \lambda\right)$. If, however, $(a)$, the sign of $F\left(\lambda_{i}+\delta \lambda\right)$ and the sign of $F\left(\lambda_{i+1}-\delta \lambda\right)$ differ, then $F_{2}$ will have at least one zero between $\lambda_{i}$ and $\lambda_{i+1}$. If, $(b)$, for every such zero $\mu_{i}$ in the interval $\left(\lambda_{i} \lambda_{i+1}\right)$ neither $F\left(\mu_{i}+\delta \lambda\right)$ nor $F\left(\mu_{i}-\delta \lambda\right)$ is zero, then the zeros $\mu_{i}$ must be isolated. Then, if $\mu_{i}$ and $\mu_{i+1}$ are successive zeros of $F_{2}$ in $\left(L_{1} L_{z}\right)$ and if, $(c)$, the signs of $F\left(\mu_{i}+\delta \lambda\right)$ and $F\left(\mu_{i+1}-\delta \lambda\right)$ differ, then $F_{1}$ will have at least one zero between the zeros of $F_{2}$. The condition $(a)$, under the original assumption that $F_{1}$ is known to vanish at a succession of values $\lambda_{1}, \ldots, \lambda_{n}$, forms an existence theorem for system II and the conditions $(a),(b)$, and $(c)$ together assert that the characteristic numbers of systems I and II separate each other.

Now we have

$$
F\left(\lambda_{i}+\delta \lambda_{)}\right)=\left(F_{2} \cdot \delta F_{1}+F_{1} \cdot \delta F_{2}\right)_{\lambda=\lambda_{i}}+\text { terms of higher order }
$$

* Bôcher, Leçons, pp. 7-8. 
and since $F_{1}\left(\lambda_{i}\right)=0$, we shall have to a first approximation

$$
F\left(\lambda_{i}+\delta \lambda\right)=\left(F_{2} \cdot \delta F_{1}\right)_{\lambda=\lambda_{i}} \text { and } F\left(\lambda_{i+1}-\delta \lambda\right)=\left(F_{2} \cdot \delta^{\prime} F_{1}\right)_{\lambda=\lambda_{i+1}}
$$

Throughout this paper $\delta^{\prime}$ will be used to denote the increment which results from a negative increment of the independent variable.

These results may now be stated in the following fundamental theorem:

Suppose $\left(\lambda_{1}, \lambda_{2}, \ldots, \lambda_{n}\right)$ are successive characteristic numbers of system I and $\left(\mu_{1}, \mu_{2}, \ldots, \mu_{n}\right)$ successive characteristic numbers of system II. If $\left(\boldsymbol{F}_{\mathbf{2}} \cdot \boldsymbol{\delta} \boldsymbol{F}_{1}\right)_{\lambda=\lambda_{i}}$ and $\left(F_{2} \cdot \delta^{\prime} F_{1}\right)_{\lambda=\lambda_{i+1}}$ have different signs, then system II has at least one characteristic number between $\lambda_{i}$ and $\lambda_{i+1}$.

If $\left(F_{1} \cdot \delta F_{2}\right)_{\lambda=\mu_{i}}$ and $\left(F_{1} \cdot \delta^{\prime} F_{2}\right)_{\lambda=\mu_{i+1}}$ also have different signs, then the characteristic numbers of systems I and II alternate.

\section{Application to Sturm's system}

The theorem developed in the last section will be applied first to the simple problem of finding conditions which will assure the existence of characteristic numbers of the following system of second order:

$$
\begin{aligned}
\frac{d}{d x}\left[k(x) u^{\prime}(x)\right]+g(\lambda, x) u(x) & =0, & a \leqq x \leqq b, \\
\alpha_{1}(\lambda) u(a)+\alpha_{2}(\lambda) u^{\prime}(a) & =0, & -\infty \leqq \lambda \leqq+\infty, \\
\beta_{1}(\lambda) u(b)+\beta_{2}(\lambda) u^{\prime}(b) & =0 . &
\end{aligned}
$$

We shall assume $\alpha_{i}(\lambda)$ and $\beta_{i}(\lambda)$ to be continuous functions of $\lambda, k(x)$ to be continuous and positive in $(a b)$ and $g(\lambda, x)$ a continuous, monotone increasing function in both arguments with the added condition that

$$
\lim _{\lambda=-\infty} g(\lambda, x)=-\infty, \quad \lim _{\lambda=\infty} g(\lambda, x)=+\infty
$$

This condition may be shown to insure as large a number of zeros as we choose in the interval $(a b)$ for any continuous solution $u(\lambda, x)$ by taking $\lambda$ sufficiently large.* Moreover, due to the continuity conditions on the coefficients these zeros are continuous functions of $\lambda$ except, perhaps, those at one extremity or the other of $(a b) . t$

* Bôcher, Leçons, § 13.

† Bôcher, Leģons, p. 61. 
Since there exists a solution of (1) which satisfies for all values of $\lambda$ the initial conditions $u(a)=\alpha_{2}, u^{\prime}(a)=-\alpha_{1}$, where $\left|\alpha_{1}\right|+\left|\alpha_{2}\right| \neq 0$, it is clear that for every value of $\lambda$ there exists a solution which satisfies (1) and the first condition of (2). Assume, now, that $\alpha_{1} / \alpha_{2}$ is a non-decreasing function of $\lambda$. Clearly, then, $\alpha_{2}$ could not vanish unless $\alpha_{1}$ vanished also, but this case has already been excluded. Consequently, since $u(\lambda, x)$ will vanish in $(a b)$ as many times as we please by choosing $\lambda$ sufficiently large and since these zeros are continuous functions of $\lambda$, the zeros must enter the $(a b)$ interval through $b$. Hence $u(\lambda, b)$ will vanish at an infinite number of values of $\lambda$, i. e. $\left(\lambda_{1}, \lambda_{2}, \ldots\right)$. In the special case $\alpha_{2} \equiv 0$, no special assumption needs to be made since the zeros must necessarily enter through either $x=a$ or $x=b$.

Hence we see that for an infinite number of values solutions exist for the auxiliary system

$$
\begin{aligned}
& \frac{d}{d x}\left[k(x) u^{\prime}(x)\right]+g(\lambda, x) u(x)=0, \\
& \alpha_{1} u(a)+\alpha_{2} u^{\prime}(a)=0, \\
& u(b)=0 .
\end{aligned}
$$

In order to apply the theorem of the last section we must consider the characteristic functions $F_{1}(\lambda)$ and $F_{8}(\lambda)$, where the first belongs to the auxiliary system just written down and the second to system (1), (2).

It is clear that we may, without loss of generality, specialize a fundamental system of integrals $u_{1}(x), u_{2}(x)$ by means of the relations

$$
\begin{array}{ll}
u_{1}(b)=0, & \beta_{1} u_{1}(b)+\beta_{2} u_{1}^{\prime}(b)=1, \\
u_{2}(b)=1, & \beta_{1} u_{2}(b)+\beta_{2} u_{2}^{\prime}(b)=0 .
\end{array}
$$

In terms of these integrals the characteristic functions are easily seen to take the following simple form:

$$
F_{1}=\alpha_{1} u_{1}(a)+\alpha_{2} u_{1}^{\prime}(a), \quad F_{2}=-\left[\alpha_{1} u_{2}(a)+\alpha_{2} u_{2}^{\prime}(a)\right] .
$$

Since, by hypothesis.

$$
F_{1}\left(\lambda_{i}\right)=0,
$$

we shall have

$\left(\delta F_{1} F_{2}\right)_{\lambda=\lambda_{i}}=-\left(\alpha_{1} \delta u_{1}+\alpha_{2} \delta u_{1}^{\prime}+u_{1} \delta \alpha_{1}+u_{1}^{\prime} \delta \alpha_{2}\right)\left(\alpha_{1} u_{2}+\alpha_{2} u_{2}^{\prime}\right)_{\lambda=\lambda_{i}}$. 
By means of the method of variation of parameters it may be shown that

$$
\begin{aligned}
& \delta u_{i}^{(j)}(x)= P_{i} u_{1}^{(j)}(x)+Q_{i} u_{2}^{(j)}(x) \\
&-\frac{\beta_{2}}{k(b)} \int_{b}^{x} \delta g(t) u_{i}(t)\left[u_{2}(t) u_{1}^{(j)}(x)-u_{1}(t) u_{2}^{(j)}(x)\right] d t \\
& \quad(i=1,2 ; j=0,1)
\end{aligned}
$$

where we have set for brevity

$$
\begin{aligned}
P_{i} & =-\beta_{2}\left[\delta u_{i}(b) u_{2}^{\prime}(b)-\delta u_{i}^{\prime}(b) u_{2}(b)\right], \\
Q_{i} & =-\beta_{2}\left[\delta u_{i}^{\prime}(b) u_{1}(b)-\delta u_{i}(b) u_{1}^{\prime}(b)\right] .
\end{aligned}
$$

Consequently, making use of (5) and noting that $Q_{1}=0$, we have

$\left(\delta F_{1} F_{2}\right)_{\lambda=\lambda_{i}}=\frac{\beta_{2}}{k(b)}\left[\int_{a}^{b} \delta g u_{1}^{2} d t\left(\alpha_{1} u_{2}+\alpha_{2} u_{2}^{\prime}\right)^{2}+\left(\frac{k(b)}{\beta_{2}}\right)^{2}\left(\frac{\alpha_{2}^{2}}{k(a)}\right) \delta\left(\frac{\alpha_{1}}{\alpha_{2}}\right)\right]$.

Similarly

$$
\begin{aligned}
\left(\delta F_{2} F_{1}\right)_{\lambda=\mu_{i}}=-\frac{\beta_{2}}{k(b)}\left\{\left[-k(b) \delta\left(\frac{\beta_{1}}{\beta_{2}}\right)+\int_{a}^{b} \delta g u_{2}^{2} d t\right]\left(\alpha_{1} u_{1}+\alpha_{2} u_{1}^{\prime}\right)^{2}\right. & \left.+\left(\frac{k(b)}{\beta_{2}}\right)^{2}\left(\frac{\alpha_{2}^{2}}{k(a)}\right) \delta\left(\frac{\alpha_{1}}{\alpha_{2}}\right)\right\} .
\end{aligned}
$$

From these two expressions, recalling the fundamental theorem, we may conclude that the characteristic numbers of the two systems will alternate if the following conditions are fulfilled:

(a) $g(\lambda, x)$ is a monotone increasing function of $x$ and $\lambda$, satisfying condition (3);

(b) $\beta_{2} / \beta_{1}$ and $\alpha_{1} / \alpha_{2}$ are non-decreasing functions of $\lambda$;

(c) $\left|\beta_{2}\right|$ is bounded below;

(d) $\left|\alpha_{1}\right|+\left|\alpha_{8}\right| \neq 0$.

These conditions are the well known conditions for the existence of characteristic numbers for the Sturmian system (1), (2).

III. The General SELF ADJOINT SYSTEM OF SECOND ORDER

We shall consider next the general self adjoint system of second order, 


$$
\begin{gathered}
\frac{d}{d x}\left[k(x) u^{\prime}(x)\right]+g(\lambda, x) u(x)=0, \\
\alpha_{11}(\lambda) u(a)+\alpha_{12}(\lambda) u^{\prime}(a)=\beta_{11}(\lambda) u(b)+\beta_{12}(\lambda) u^{\prime}(b), \\
\alpha_{21}(\lambda) u(a)+\alpha_{22}(\lambda) u^{\prime}(a)=\beta_{21}(\dot{\lambda}) u(b)+\beta_{22}(\lambda) u^{\prime}(b), \\
k(a)\left(\beta_{11} \beta_{22}-\beta_{21} \beta_{12}\right)=k(b)\left(\alpha_{11} \alpha_{22}-\alpha_{21} \alpha_{12}\right) .
\end{gathered}
$$

We shall suppose that $k(x)$ and $g(\lambda, x)$ are limited by the same conditions as were imposed upon them in the last section. If now we let

$L_{i j}=L_{i}\left(u_{j}\right)=\alpha_{i 1} u_{j}(a)+\alpha_{i 2} u_{j}^{\prime}(a), \quad M_{i j}=M_{i}\left(u_{j}\right)=\beta_{i 1} u_{j}(b)+\beta_{i 2} u_{j}^{\prime}(b)$,

where $u_{j}$ is any particular solution of the differential equation, and choose for an auxiliary system the Sturmian system whose boundary conditions are

$$
L_{1}(u)=0, \quad M_{1}(u)=0,
$$

then the functions $F_{1}$ and $F_{2}$ will be defined as follows:

$$
F_{1}(\lambda)=\left|\begin{array}{ll}
L_{11} & L_{12} \\
M_{11} & M_{12}
\end{array}\right|, \quad F_{2}(\lambda)=\left|\begin{array}{l}
L_{11}-M_{11}, L_{12}-M_{12} \\
L_{21}-M_{21}, L_{22}-M_{22}
\end{array}\right|
$$

Let

$$
\begin{aligned}
L_{11}=0, & L_{21}=1, \\
L_{12}=1, & L_{22}=0, \\
\beta_{11} \beta_{22}-\beta_{21} \beta_{12}=-k(b), & \alpha_{11} \alpha_{22}-\alpha_{21} \alpha_{12}=-k(a) .
\end{aligned}
$$

It is always possible to do this since the first conditions merely particularize a fundamental set of solutions $\left(u_{1}\right.$ and $\left.u_{2}\right)$, while the last condition amounts to multiplying the boundary conditions by a suitably chosen function of $\lambda$.

From these conditions, since $u_{1} u_{2}^{\prime}-u_{2} u_{1}^{\prime}=1 / k(x)$, it follows that

$$
M_{11} M_{28}-M_{12} M_{21}=-1
$$

Consequently

$$
\begin{aligned}
& F_{1}(\lambda)=-M_{11}, \\
& F_{2}(\lambda)=M_{18}+M_{21}-2,
\end{aligned}
$$


whence

$$
\left(\delta F_{1} F_{2}\right)_{\lambda=\lambda_{i}}=-\delta M_{11}\left(M_{12}+M_{21}-2\right)_{\lambda=\lambda_{i}} .
$$

By means of the variation of parameters, it may be shown that $\delta u_{i}^{(j)}(x)=P_{i} u_{1}^{(j)}(x)+Q_{i} u_{2}^{(j)}(x)-\int_{a}^{x} \delta g(t) u_{i}(t)\left[u_{1}(t) u_{2}^{(j)}(x)-u_{2}(t) u_{1}^{(j)}(x)\right] d t$
$(i=1,2 ; j=0,1)$, where we have set for brevity

$$
\begin{aligned}
P_{i} & =k(a)\left[\delta u_{i}(a) u_{2}^{\prime}(a)-\delta u_{i}^{\prime}(a) u_{2}(a)\right], \\
Q_{i} & =k(a)\left[\delta u_{i}^{\prime}(a) u_{1}(a)-\delta u_{i}(a) u_{1}^{\prime}(a)\right] .
\end{aligned}
$$

Since $\left(M_{11}\right)_{\lambda=\lambda_{i}}=0$ and since, from the self adjoint condition on the coefficients

$$
\alpha_{11} \delta \alpha_{22}+\alpha_{22} \delta \alpha_{11}-\alpha_{21} \delta \alpha_{12}-\alpha_{12} \delta \alpha_{21}=0 \text {, }
$$

$$
\beta_{11} \delta \beta_{22}+\beta_{22} \delta \beta_{11}-\beta_{21} \delta \beta_{12}-\beta_{12} \delta \beta_{21}=0,
$$

we have

$$
\begin{array}{r}
-\left(\delta F_{1} F_{2}\right)_{\lambda=\lambda_{i}}=\left(Q_{1} M_{12}-\int_{a}^{b} \delta g u_{1}^{2} d t M_{12}\right)\left(M_{12}+M_{21}-2\right)+\delta \beta_{11}\left(\frac{\beta_{12}}{k(b)}\right. \\
\left.+\beta_{21} u_{1}^{2}+\beta_{22} u_{1} u_{1}^{\prime}-2 u_{1}\right)+\delta \beta_{12}\left(-\frac{\beta_{11}}{k(b)}+\beta_{21} u_{1} u_{1}^{\prime}+\beta_{22} u_{1}^{\prime 2}-2 u_{1}^{\prime}\right) .
\end{array}
$$

Let us recall from (8), since $M_{11}=0$, that $M_{12} M_{21}=1$. Hence

$(10)-\left(\delta F_{1} F_{2}\right)_{\lambda=\lambda_{i}}=\left(Q_{1}-\int_{a}^{b} \delta g u_{1}^{2} d t\right)\left(M_{12}-1\right)^{2}+k(b) \delta\left(\frac{\beta_{11}}{\beta_{12}}\right)\left(u_{1}-\frac{\beta_{12}}{k(b)}\right)^{2}$

Similarly

$$
-\left(\delta F_{2} F_{1}\right)_{\lambda=\mu_{i}}=\left(\delta M_{12}+\delta M_{21}\right) M_{11}
$$

Since, from (7) and (9), we get $P_{1}+Q_{8}=0$, and since

$$
F_{2}\left(\mu_{i}\right)=\left(M_{12}+M_{21}-2\right)_{\lambda=\mu_{i}}=0
$$


it follows that

$$
\begin{aligned}
-\left(\delta F_{2} F_{1}\right)_{\lambda=\mu_{i}} & =\left(P_{2}-\int_{22}\right) M_{11}^{2}+2\left(Q_{2}+\int_{21}\right) M_{11}\left(M_{12}-1\right) \\
& -\left(Q_{1}+\int_{11}\right)\left(1-M_{12}\right)^{2}+\left(u_{2} \delta \beta_{11}+u_{2}^{\prime} \delta \beta_{12}+u_{1} \delta \beta_{21}+u_{1}^{\prime} \delta \beta_{22}\right) M_{11},
\end{aligned}
$$

where we have used the abbreviation $\int_{i j}=\int_{a}^{b}-\delta g u_{i} u_{j} d t$.

But by the use of (8) and (11) and the fact that $u_{1} u_{2}^{\prime}-u_{2} u_{1}^{\prime}=1 / k(x)$, it may be shown that

$$
\begin{aligned}
\left(u_{2} \delta \beta_{11}+u_{2}^{\prime} \delta\right. & \left.\beta_{12}+u_{2} \delta \beta_{21}+u_{1}^{\prime} \delta \beta_{22}\right) M_{11}=\left(\beta_{11} \delta \beta_{21}\right. \\
- & \left.-\beta_{21} \delta \beta_{11}\right) u_{1}^{2}+2\left(\beta_{12} \delta \beta_{21}-\beta_{22} \delta \beta_{11}\right) u_{1} u_{1}^{\prime}+\left(\beta_{12} \delta \beta_{22}\right. \\
& \left.\quad-\beta_{22} \delta \beta_{12}\right) u_{1}^{\prime 2}+2 \delta \beta_{11} u_{1}+2 \delta \beta_{12} u_{1}^{\prime}+\left(\beta_{11} \delta \beta_{12}-\beta_{12} \delta \beta_{11}\right) / k(b) .
\end{aligned}
$$

In order to show that this quadratic polynomial is always positive, it will be sufficient to know that $D_{3}$, the determinant of the coefficients, is positive or zero and that $D_{2}$ and $D_{1}$, the leading principal minors of the determinant, are both positive. Similarly the polynomial will be negative if $D_{3}$ is negative or zero, $D_{2}$ positive, and $D_{1}$ negative.

By use of the second equation in (9) and by a proper combination of multiples of columns in $D_{3}$, it follows readily that

$$
D_{1}=\beta_{11} \delta \beta_{21}-\beta_{21} \delta \beta_{11}, \quad D_{2}=k(b)\left(\delta \beta_{12} \delta \beta_{21}-\delta \beta_{22} \delta \beta_{11}\right), \quad D_{3}=0
$$

Similarly the determinant of the quadratic form in $M_{11}$ and $\left(M_{12}-1\right)$ is

$$
\begin{aligned}
D_{4} & =\left(Q_{2}+\int_{21}\right)^{2}+\left(P_{2}-\int_{11}\right)\left(Q_{1}+\int_{11}\right) \\
& =Q_{2}^{2}+P_{2} Q_{1}+\int_{a}^{b}-\delta g\left(-Q_{1} u_{2}^{2}+2 Q_{2} u_{1} u_{2}+P_{2} u_{1}^{2}\right) d t+\int_{21}^{2}-\int_{11} \int_{22} .
\end{aligned}
$$


From these determinants and from (10), it is evident that the theorem of the first section will apply providing the following conditions are imposed, in which, as before, the prime pertains to the increment resulting from a negative increment of the independent variable:

At

$$
\begin{array}{ll}
\lambda=\lambda_{i} ; & Q_{1}<0, \quad \delta\left(\frac{\beta_{12}}{\beta_{11}}\right) \geqq 0, \\
\lambda=\lambda_{i+1} ; & Q_{1}^{\prime}>0, \quad \delta^{\prime}\left(\frac{\beta_{12}}{\beta_{11}}\right) \leqq 0,
\end{array}
$$

$$
\begin{aligned}
& \lambda=\mu_{i} ; \quad Q_{2}^{2}+P_{2} Q_{1} \leqq 0, \quad Q_{1}<0, \\
& \delta\left(\frac{\beta_{21}}{\beta_{11}}\right) \geqq 0, \quad \delta \beta_{11} \delta \beta_{22}-\delta \beta_{21} \delta \beta_{12} \leqq 0 \\
& \lambda=\mu_{i+1} ; Q_{2}^{\prime 2}+P_{2}^{\prime} Q_{1}^{\prime} \leqq 0, \quad Q_{1}^{\prime}>0, \\
& \delta^{\prime}\left(\frac{\beta_{21}}{\beta_{11}}\right) \leqq 0, \quad \delta^{\prime} \beta_{11} \delta^{\prime} \beta_{22}-\delta^{\prime} \beta_{21} \delta^{\prime} \beta_{12} \leqq 0
\end{aligned}
$$

Since $Q_{2}^{2}+P_{2} Q_{1}=(1 / k(a))\left(\delta \alpha_{22} \delta \alpha_{11}-\delta \alpha_{12} \delta \alpha_{21}\right)$ and $Q_{1}=\left(\alpha_{11}^{2} / k(a)\right)$ . $\delta\left(\alpha_{18} / \alpha_{11}\right)$, we may state the following existence theorems:

TheOREM I. Between two successive characteristic numbers of the Sturmian system $L_{1}=0, M_{1}=0$, there must always lie at least one characteristic number for the general self adjoint system of second order $L_{1}=M_{1}, L_{2}=M_{2}$.

THEOREM II. Under the additional conditions

$$
\begin{aligned}
& \frac{\beta_{21}}{\beta_{11}} \text { a non-decreasing function, }\left|\beta_{11}\right|+\left|\beta_{21}\right| \neq 0 \\
& \delta \beta_{11} \delta \beta_{22}-\delta \beta_{21} \delta \beta_{12} \text { and } \delta^{\prime} \beta_{11} \delta^{\prime} \beta_{22}-\delta^{\prime} \beta_{21} \delta^{\prime} \beta_{12} \leqq 0 \\
& \delta \alpha_{11} \delta \alpha_{22}-\delta \alpha_{21} \delta \alpha_{12} \text { and } \delta^{\prime} \alpha_{11} \delta^{\prime} \alpha_{22}-\delta^{\prime} \alpha_{21} \delta^{\prime} \alpha_{12} \leqq 0
\end{aligned}
$$

the characteristic numbers of the Sturmian system and the general self adjoint system alternate.

It must be noted that both of these theorems are valid only under the assumption that the rank of the matrix of the general system is different from zero. This exception is the problem treated in the next section. 


\section{The case of double values}

We observe, first, that when $M_{11}=0, M_{22}=0, M_{12}=1, M_{21}=1$, the rank of the matrix $T_{2}$ (Section $I$ ) is zero and consequently two linearly independent solutions must exist for such a value of $\lambda$. Let $\mu$ be such a double value of $\lambda$.

Then

$$
\begin{aligned}
& (\delta F)_{\lambda=\mu}=0, \quad\left(\delta^{2} F\right)_{\lambda=\mu}=0, \quad\left(\delta^{3} F\right)_{\lambda=\mu}=3\left(\delta F_{1} \delta^{2} F_{2}\right)_{\lambda=\mu}, \\
& \left(\delta F_{1}\right)_{\lambda=\mu}=-\left(\delta M_{11}\right)_{\lambda=\mu}=-Q_{1}+\int_{a}^{b} \delta g u_{1}^{2} d t+\frac{\beta_{12}^{2}}{k(b)} \delta\left(\frac{\beta_{12}}{\beta_{11}}\right) .
\end{aligned}
$$

Consequently, under the conditions already imposed, $F_{1}$ changes sign at $\mu$. We next compute

$$
\begin{aligned}
\left(\delta^{2} F_{2}\right)_{\lambda=\mu} & =2\left(\delta M_{22} \delta M_{11}-\delta M_{12} \delta M_{21}\right)_{\lambda=\mu} \\
& =2\left(\sum_{i=1}^{6} A_{i} U_{i}\right)
\end{aligned}
$$

where the $A_{i}$ are the determinants in order that can be formed from the matrix

$$
\left\|\begin{array}{llll}
\beta_{11} & \beta_{12} & \delta \beta_{11} & \delta \beta_{12} \\
\beta_{21} & \beta_{22} & \delta \beta_{21} & \delta \beta_{22}
\end{array}\right\|
$$

and the $U_{i}$ are the corresponding determinants from the matrix

$$
\left\|\begin{array}{llll}
\delta u_{7} & \delta u_{1}^{\prime} & u_{1} & u_{1}^{\prime} \\
\delta u_{2} & \delta u_{2}^{\prime} & u_{2} & u_{2}^{\prime}
\end{array}\right\|
$$

From a computation based on the formulas of the last section it may be proved that

$$
U_{1}=-\frac{1}{k(b)} D_{4} ; \quad U_{6}=\frac{1}{k(b)}
$$


Upon expansion $A_{2} U_{2}+A_{3} U_{3}+A_{4} U_{4}+A_{5} U_{5}$ is found to be a quadratic form in $u_{1}, u_{2}, u_{1}^{\prime}, u_{2}^{\prime}$ whose matrix is the following symmetric array:

$$
\left\|\begin{array}{llll}
A_{2} K & A_{3} K & A_{2} M & A_{3} M \\
A_{3} K & A_{5} K & A_{3} M & A_{5} M \\
A_{2} M & A_{3} M & A_{2} N & A_{3} N \\
A_{3} M & A_{5} M & A_{3} N & A_{5} N
\end{array}\right\|
$$

where

$K=-P_{2}-\int_{a}^{b} \delta g u_{2}^{2} d t, M=-Q_{2}+\int_{a}^{b} \delta g u_{1} u_{2} d t, N=Q_{1}-\int_{a}^{b} \delta g u_{1}^{2} d t$.

'Suppose $\Delta_{4}$ is the determinant of the matrix and $\Delta_{1}, \Delta_{2}, \Delta_{3}$ are the leading principal minors of $\Delta_{4}$. Then the quadratic form will be positive definite providing $\Delta_{1}, \ldots, \Delta_{4}$ are all positive, or negative definite if $\Delta_{1}, \ldots, \Delta_{4}$ are alternately negative and positive.

It can be proved upon expansion that $\Delta_{1}, \ldots, \Delta_{4}$ are resolvable into the following factors:

$$
\begin{array}{ll}
\Delta_{1}=A_{2} K, & \Delta_{3}=A_{2} K\left(A_{2} A_{5}-A_{8}^{2}\right)\left(K N-M^{2}\right), \\
\Delta_{2}=K^{2}\left(A_{2} A_{5}-A_{3}^{2}\right), & \Delta_{4}=\left(A_{2} A_{5}-A_{8}^{2}\right)^{2}\left(K N-M^{2}\right)^{2} .
\end{array}
$$

Consequently we must demand that

$$
A_{2} A_{5}-A_{8}^{2} \geqq 0, \quad K N-M^{2} \geqq 0
$$

and the sign of the form will be that of $A_{2} K$.

But

$$
\begin{aligned}
K N-M^{2} & =-D_{4} \text { (of the preceding section), } \\
A_{2} A_{5}-A_{8}^{2} & =D_{2} \\
A_{2} & =D_{1} .
\end{aligned}
$$

Therefore, since it follows from (12) that $\dot{A}_{2} K \leqq 0$, the quadratic form must be negative definite under the restrictions imposed by Theorem II, from which it follows that $\delta^{2} F_{2}<0$. 
Theorem III. Under the conditions of Theorem II, two successive zeros of $F_{9}$ are always separated by a zero of $F_{1}$. As a limiting case two zeros of $F_{2}$ may coincide with a zero of $F_{1}$. Since the sign of $F=F_{1} F_{2}$ changes at every zero of $F_{1}$ and $F_{2}$, the ordering of the zeros is uniquely determined from a knowledge of the sign of $F$ when $\lambda$ approaches negative infinity.

\section{Extension to THE CASE Where $k$ VARIES WITH $\lambda$}

The conclusions just obtained afford an easy method of extension to the case where $k$ is a function of $\lambda$ as well as of $x$.

If we transform the general system (6) by means of the transformation $u=Q(x) v$ where $Q(x)=1 / \sqrt{k(x)}$, we shall obtain the following system

$$
\begin{gathered}
v^{\prime \prime}+q(\lambda, x) v=0 \\
A_{11} v(a)+A_{12} v^{\prime}(a):=B_{11} v(b)+B_{12} v^{\prime}(b) \\
A_{21} v(a)+A_{22} v^{\prime}(a)=B_{21} v(b)+B_{22} v^{\prime}(b)
\end{gathered}
$$

where $q(\lambda, x)=Q\left[\left(k Q^{\prime}\right)^{\prime}+g Q\right]$, and

$$
\begin{array}{ll}
A_{i 1}=\alpha_{i 1} Q(a)+\alpha_{i 2} Q^{\prime}(a), & A_{i 2}=\alpha_{i 2} Q(a), \\
B_{i 1}=\beta_{i 1} Q(b)+\beta_{i 2} Q^{\prime}(b), & B_{i 2}=\beta_{i 2} Q(b) \quad(i=1,2) .
\end{array}
$$

A simple computation will show that the new system is also self adjoint.

Now the values of $\lambda$ which furnish a solution of this system are evidently values which furnish a solution of system (6) since we may pass from one to the other by means of a non-singular transformation.

But system (13) is itself included under the theorems which apply to system (6) so that if the coefficients of (13) are properly restricted the existence of $\lambda$ 's for this system and consequently for system (6) will be established. Since the $k$ is now absorbed in $q(\lambda, x)$ we need no longer restrict it to be a function independent of $\lambda$.

We should notice also that, since $Q$ does not vanish in the interval, the oscillation properties of $u$ and $v$ are identical.

\section{The ORDERING OF THE zeros OF $F_{1}$ AND $F_{2}$}

We saw from Theorem $I I$ that the ordering of the zeros of $F_{1}$ and $F_{2}$ is uniquely determined from a knowledge of the sign of $F$ when $\lambda$ approaches 
negative infinity. We consider $F=F_{1} F_{2}=-M_{11} M_{12}-M_{11} M_{21}+2 M_{11}$ as $\lambda \rightarrow-\infty$.

From the relation $u_{1} u_{2}^{\prime}-u_{2} u_{1}^{\prime}=1 / k(x)$ and recalling (7) we may prove that

$$
\begin{aligned}
& u_{2}(b)=\left[-\frac{\alpha_{22}}{\alpha_{12}}+\int_{a}^{b} \frac{d t}{\left.k(t) \frac{d t}{u_{1}^{2}(t)}\right] u_{1}(b)}=S u_{1}(b),\right. \\
& u_{2}^{\prime}(b)=\left[-\frac{\alpha_{22}}{\alpha_{12}}+\int_{a}^{b} \frac{d t}{k(t) u_{1}^{2}(t)}\right] u_{1}^{\prime}(b)+\frac{1}{k(b) u_{1}(b)}=S u_{1}^{\prime}(b)+T .
\end{aligned}
$$

Under the conditions already imposed upon $u_{1}$ it may be shown* that for $x>a$,

$$
\lim _{\lambda=-\infty} \frac{u_{1}^{\prime}(x)}{u_{1}(x)}=\infty, \quad \lim _{\lambda=-\infty} u_{1}^{\prime}(x)=\infty, \quad \lim _{\lambda=-\infty} u_{1}(x)=\infty .
$$

Substituting the values for $u_{2}(b)$ and $u_{2}^{\prime}(b)$ in $F$ we have

$$
\begin{aligned}
F=-\left[\left(\beta_{11}^{2} S+\beta_{11} \beta_{21}\right) u_{1}^{2}+\left(\beta_{11} \beta_{22}+\beta_{12} \beta_{21}+2 \beta_{11} \beta_{12} S\right) u_{1} u_{1}^{\prime}\right. \\
\left.\quad+\left(\beta_{12}^{2} S+\beta_{12} \beta_{22}\right) u_{1}^{\prime 2}+\left(\beta_{11} \beta_{12} T^{\prime}-2 \beta_{11}\right) u_{1}+\left(\beta_{12}^{2} T-2 \beta_{12}\right) u_{1}^{\prime}\right] .
\end{aligned}
$$

If we notice that the discriminant of the first three terms of $F$, since $\beta_{21} \beta_{12}-\beta_{11} \beta_{22}=k(b)$, is equal to $k^{2}(b)$, it may be verified that $F$ is factorable as follows:

$$
F=-\pi[B \pi+k(b)]\left[u_{1}-\frac{\beta_{12}+\beta_{12} \sqrt{\frac{-B \pi}{k(b)}}}{B \pi+k(b)}\right]\left[\begin{array}{r}
\beta_{12}+\beta_{12} \sqrt{\frac{-B \pi}{k(b)}} \\
B \pi+k(b)
\end{array}\right],
$$

where $B=\beta_{12}^{2} S+\beta_{12} \beta_{21}$, and $\pi=u_{1}^{\prime} / u_{1}+\beta_{11} / \beta_{12}$.

If we make the restriction that $\beta_{11}$ and $\beta_{12}$ shall be bounded when $\lambda \rightarrow-\infty$, recalling from Section $I$ that $\beta_{12}$ can never vanish, we see that the sign of $F$ depends entirely upon the sign of $B$, being positive when $B<0$, and negative when $B \geqq 0$.

* Bôcher, Leçons, pp. 65-66. 
It is important to notice that we have here assumed that $\alpha_{12} \neq 0$, but if this were not the case then we could express $u_{1}(b)$ in terms of $u_{2}(b)$, choose for our comparison system $\boldsymbol{F}=\boldsymbol{M}_{\mathbf{2 2}}$, and obtain a similar result.

These results, recalling Theorems II and III, may be stated as follows:

Theorem IV. If $\beta_{11}$ and $\beta_{12}$ are bounded as $\lambda$ approaches negative infinity, then the first characteristic number of the Sturmian system precedes the first characteristic number of the general self adjoint system provided $\lim _{\lambda=-\infty} B \geqq 0$; but the first characteristic number of the general system precedes or (in the case of double values) coincides with the first characteristic number of the Sturmian system provided $\lim _{\lambda=-\infty} B<0$.

INDIANA UNIVERSITY, Bloomington, Ind. 